\title{
Estimates and bounds on the rate and extent of allele frequency change
}

\author{
DAVID W. TONKYN \\ Department of Biological Sciences, Clemson University, Clemson, South Carolina, 29634 U.S.A.
}

\begin{abstract}
Two approximations are provided to the frequency of an autosomal allele after any number of generations of selection and, conversely, to the number of generations required for any given frequency change. These estimates are accurate over a wide range of selection coefficients and initial conditions, and have the added advantage of providing strict upper and lower bounds to the true values under quite general conditions. The approach employed here, of finding exact solutions to approximating difference equations, could easily be applied to models with migration, mutation, etc. and, indeed, to any non-linear difference equation. In such applications, it offers several advantages over traditional methods from approximation theory.
\end{abstract}

Keywords: allele frequencies, approximations, modelling, population genetics, selection.

\section{Introduction}

One of the basic questions of microevolutionary theory is how do allele frequencies change through time in response to selection and other forces. Haldane first addressed this question in a series of papers on singlelocus models, assuming various mating systems and inheritance patterns (Haldane, 1932a, for review). Much of the current research in population genetics remains focused on this question, although the models now include the complicating effects of, for example, multi-locus interactions, spatially varying selection regimes, and population dynamics (e.g. Feldman, 1989). Haldane found that even the simplest models for populations with discrete generations often yielded 'formidable' non-linear difference equations whose solutions could only be approximated. The accuracy of these approximations varies, depending on the allele frequencies and parameter values. Of course, where greater accuracy is needed, the original equations can now be solved numerically by computer. Nevertheless, it is often useful to have simple, approximate solutions, as a check on the numerical solutions and, more importantly, as components in larger genetics models such as those incorporating population dynamics.

This paper presents simple, explicit estimates on the rate and extent of change in the frequency of an autosomal allele under selection. These estimates are extremely accurate over a wide range of selection coefficients and initial conditions, and have the added advantage of providing strict upper and lower bounds to the true values under fairly general conditions. They may rival in accuracy and utility the more complex approximations by Haldane (1932a) and Wright (1931) under certain conditions. The approach employed here, of finding exact solutions to approximating difference equations, could easily be applied to models with migration, mutation, and so on.

Consider a large, panmictic population of sexually reproducing organisms, with discrete generations. Suppose that $A_{1}$ and $A_{2}$ are two alleles at an autosomal locus which occur at frequencies $p$ and $1-p$. Suppose also that the relative fitnesses of the three genotype $\mathrm{A}_{1} \mathrm{~A}_{1}, \mathrm{~A}_{1} \mathrm{~A}_{2}$ and $\mathrm{A}_{2} \mathrm{~A}_{2}$ are, respectively, $1, r_{1}$, and $r_{2}$. Then, in the absence of other factors, selection will change the frequency of $\mathrm{A}_{1}$ from $p_{0}$ to $p_{1}$ in one generation, where

$p_{1}=\frac{p_{0}^{2}+r_{1} p_{0}\left(1-p_{0}\right)}{p_{0}^{2}+2 r_{1} p_{0}\left(1-p_{0}\right)+r_{2}\left(1-p_{0}\right)^{2}}$.

The problem is to find $p_{n}$, the frequency of $\mathrm{A}_{1}$ alleles after $n$ generations, as a function of $p_{0}, r_{1}, r_{2}$ and $n$. Alternatively, one can ask how many generations, $n$, are required for the frequency of $A_{1}$ alleles to change from $p_{0}$ to $p_{n}$, given $r_{1}$ and $r_{2}$.

Equation (1) has been solved in closed form for only two special cases. First, if the homozygote $\mathrm{A}_{2} \mathrm{~A}_{2}$ has zero fitness $\left(r_{2}=0\right)$, then $p_{n} /\left(1-p_{n}\right)=1+\left(1 / r_{1}\right)\left(p_{n-1}\right) /$ 
$\left(1-p_{n-1}\right)$ exactly, which yields

$p_{n}=\frac{\left(1-r_{1}\right) p_{0}+\left(1-p_{0}\right) r_{1}\left(1-r_{1}^{n}\right)}{1-r_{1}+\left(1-p_{0}\right)\left(2 r_{1}-1\right)\left(1-r_{1}^{n}\right)}$

The solution has been discovered several times (see $\mathrm{Li}$, 1976, pp. 413-414). In the limit $r_{1} \rightarrow 1$ (using l'Hopital's rule, $\mathrm{Li}, 1976)$, it reduces to $p_{n}=\left[p_{0}+n\right.$ $\left.\left(1-p_{0}\right)\right] /\left[1+n\left(1-p_{0}\right)\right]$. In addition, an exact solution is known when the $A_{1}$ alleles have multiplicative effects on fitness so that the effect of two doses, relative to none, is the square of the effect of one dose: $1 / r_{2}=\left\langle r_{1}\right\rangle$ $\left.r_{2}\right)^{2}(\mathrm{Li}, 1959$, and see below).

When the selection coefficients do not satisfy either condition, then one can always solve equation (1) numerically by iteration. This will yield a value for $p_{n}$ or $n$ that is correct, but which may be tedious to obtain and which reveals nothing of its dependence on the parameters and initial conditions. A more satisfying approach is to use closed form approximations which may sacrifice little in accuracy in exchange for a greater ease of use and transparency. This paper presents two such approximations for the case of an autosomal allele under selection, and illustrates a general approach that can be applied more broadly to population genetics models with discrete generations and, indeed, to any non-linear difference equations.

\section{Approximations to $p_{n}$}

Equation (1) can be rewritten

$p_{1}=\frac{r_{1} p_{0}+\left(1-r_{1}\right) p_{0}^{2}}{r_{1} p_{0}+r_{2}\left(1-p_{0}\right)+\left(r_{1}-r_{2}\right) p_{0}\left(1-p_{0}\right)+\left(1-r_{1}\right) p_{0}^{2}}$.

If the $\mathrm{A}_{1}$ allele is dominant, then $\left(1-r_{1}\right) p_{0}^{2}$ vanishes; if $\mathrm{A}_{2}$ is dominant, $\left(r_{1}-r_{2}\right) p_{0}\left(1-p_{0}\right)$ disappears. More generally, if $p_{0}$ is small, and $r_{1}$ and $r_{2}$ are not enormously different in value, then these terms are much smaller than the leading terms in the ratio and can be set equal to zero. This yields an approximation $u_{1}$ to $p_{1}$

$u_{1}=\frac{p_{0}}{p_{0}+R\left(1-p_{0}\right)}$,

where $R=r_{2} / r_{1}$, provided the $\mathrm{A}_{2}$ allele is neither fully dominant $\left(r_{1} \neq r_{2}\right)$ nor lethal $\left(r_{2} \neq 0\right)$. One can show by direct substitution that $U_{\mathrm{i}}=u_{\mathrm{i}} /\left(1-u_{\mathrm{i}}\right)$ obeys the recursion relation $U_{\mathrm{n}}=U_{\mathrm{n}-1} / R=U_{0} / R^{\mathrm{n}}$ which on rearrangement yields

$u_{\mathrm{n}}=\frac{p_{0}}{p_{0}+R^{n}\left(1-p_{0}\right)}$.
This provides a simple approximation $u_{\mathrm{n}}$ to $p_{\mathrm{n}}$, the frequency of $\mathrm{A}_{1}$ alleles in generation $n$, as a function of $p_{0}$, $r_{1}, r_{2}$ and $n$.

This approximation has several useful features. First, $u_{n}$ is bounded between 0 and 1 for all $n=1,2$, $\ldots, r_{1}$ and $r_{2}>0$, and $0<p_{0}<1$. Second, it returns the exact solution in the special case that the $A_{1}$ alleles have multiplicative effects on fitness, when $r_{1}^{2}=r_{2}(\mathrm{Li}$, 1959). Finally, when $r_{1}^{2} \neq r_{2}, u_{n}$ provides not only an estimate but also a bound on the true value of $p_{n}$. In particular, $u_{1}>p_{1}$, hence $u_{n}>p_{0}$ for all $\mathrm{p}_{\mathrm{n}}$ and $n$ if and only if $r_{1}^{2}>r_{2}$. Therefore, this approximation provides a strict upper bound on the actual gene frequency $p_{n}$ after $n$ generations if $r_{1}^{2}>r_{2}$, and a strict lower bound if $r_{1}^{2}<r_{2}$.

A second approximation to equation (1) can be obtained by setting all terms in $p_{0}^{2}=0$, valid when the $A_{1}$ allele is rare. This yields

$$
l_{1}=\frac{p_{0}}{2 p_{0}+R\left(1-2 p_{0}\right)},
$$

again where $R=r_{2} / r_{1}$ and the $\mathrm{A}_{2}$ allele is neither fully dominant nor lethal. This equation can be linearized by the transformation $L_{n}=l_{n} /\left(1-2 l_{n}\right)=L_{n-1} / R=L_{0} / R^{n}$ to yield

$$
l_{n}=\frac{p_{0}}{2 p_{0}+R^{n}\left(1-2 p_{0}\right)} .
$$

This approximation is well-behaved if $p_{0}<0.5$, and converges monotonically to 0.5 or 0 depending on whether $r_{1}>r_{2}$ or $r_{1}<r_{2}$. It provides a strict lower or upper bound on $p_{1}$ according to whether $\left(r_{1}-r_{2}\right)$ $p_{0}+r_{2}\left(1-r_{1}\right)\left(1-p_{0}\right)>0$ or $<0$, respectively. For $p_{0}$ small, this condition effectively reduces to $r_{1}<1$ or $>$ 1 , independent of $r_{2}$. More generally, the condition simplifies and becomes independent of $p_{0}$ provided either allele is strictly favoured under selection. If $A_{1}$ is favoured $\left(1 \geq r_{1}>r_{2}\right)$, then $l_{1}<p_{1}$ and therefore $l_{n}<p_{n}$ for all $n$ as the two series increase to 0.5 and 1 , respectively. Conversely, if $A_{1}$ is strictly opposed $\left(1 \leq r_{1}<r_{2}\right)$, then $l_{1}>p_{1}$ hence $l_{n}>p_{n}$ as both measures decrease to 0 .

These approximations can be used separately or in concert. Several cases are considered.

One possibility is that the $A_{1}$ allele is fully dominant. Haldane considered this case to be the simplest, though perhaps the most important, of a large number' (1932b, p. 247), and he discussed it in two separate papers $\left(1924,1932 \mathrm{~b}\right.$, see below). In this case, $r_{l}=1$, and equation (1) can be written

$$
p_{1}=\frac{p_{0}}{1-\left(1-r_{2}\right)\left(1-p_{0}\right)^{2}} \text {. }
$$


The two approximations [equations (4) and (6)] correspond respectively to replacing the $\left(1-p_{0}\right)^{2}$ term by the slightly larger $\left(1-p_{0}\right)$ or smaller $\left(1-2 p_{0}\right)$. Here $u_{n}$ overestimates the actual change in $p$ each generation, and $l_{n}$ underestimates the change, regardless of whether $p$ is increasing or decreasing. Therefore, if the dominant allele is favoured under selection $\left(r_{2}<1\right)$, then its true frequency after $n$ generation is bracketed by the simple estimates $u_{n}>p_{n}>l_{n}$, as the three series converge respectively to 1,1 , and 0.5 . Conversely, if the dominant allele is deleterious $\left(r_{2}>1\right)$, then the inequalities are reversed, $u_{n}<p_{n}<l_{m}$, as all three converge to 0 .

More generally, if the $A_{1}$ allele is strictly favoured in selection such that $1 \geq r_{1}^{2}>r_{2}$ (which includes full dominance as a special case) then $u_{n}>p_{n}>l_{n}$ for all $n$ as the three series converge respectively to 1,1 and 0.5 .

Alternatively, if the $\mathrm{A}_{1}$ allele is strictly opposed such that $1 \leq r_{1}^{2}<r_{2}$, the order of the three values is reversed, $u_{n}<p_{n}<l_{n}$, as all three converge to 0 .

If the heterozygote is at a selective advantage $\left(1<r_{1}>r_{2}\right)$, then $p_{n}$ will approach a stable, intermediate value $p^{*}=\left(r_{1}-r_{2}\right) /\left(2 r_{1}-1-r_{2}\right)$. In this case, the two approximations (5) and (7) cannot reproduce the full range of behaviours of equation (1) but can only increase, since $r_{1}>r_{2}$, ultimately converging to 1 and
0.5 . However, if $A_{1}$ is initially rare, then the two approximations will increase with $p_{n}$, and provide accurate estimates of the latter until it asymptotes at $\mathrm{p}^{*}$. In addition, $p_{n}$ will always be bounded from above by $u_{n}$ (and $\left.p^{*}\right)$, although whether $l_{n}$ simultaneously provides a lower bound on $p_{n}$ depends on the precise values of $r_{1}, r_{2}$, and $p_{0}$, and is not assured.

Alternatively, the heterozygote may be at a selective disadvantage $\left(1>r_{1}<r_{2}\right)$; here, $p^{*}$ is an unstable equilibrium, and $p_{n}$ will go to 1 or 0 depending on which allele has the initial advantage. Again, approximations (5) and (7) cannot reproduce the full range of possibilities (both always converge to 0 ) although they provide accurate approximations to $p_{n}$ when it too converges to 0 (that is, if $p_{0}<p^{*}$ ). In this case, $u_{n}$ is always a lower bound to $p_{n}$ although whether $l_{n}$ is simultaneously an upper bound is more complex.

All these applications are for $A_{1}$ rare and not fully recessive. As alleles can be labelled arbitrarily, the approximations can be used whenever the rarer allele, whichever it is, is not fully recessive.

\section{Accuracy}

Figure 1 illustrates the accuracy of these two approximations. At the upper left is a contour plot showing the
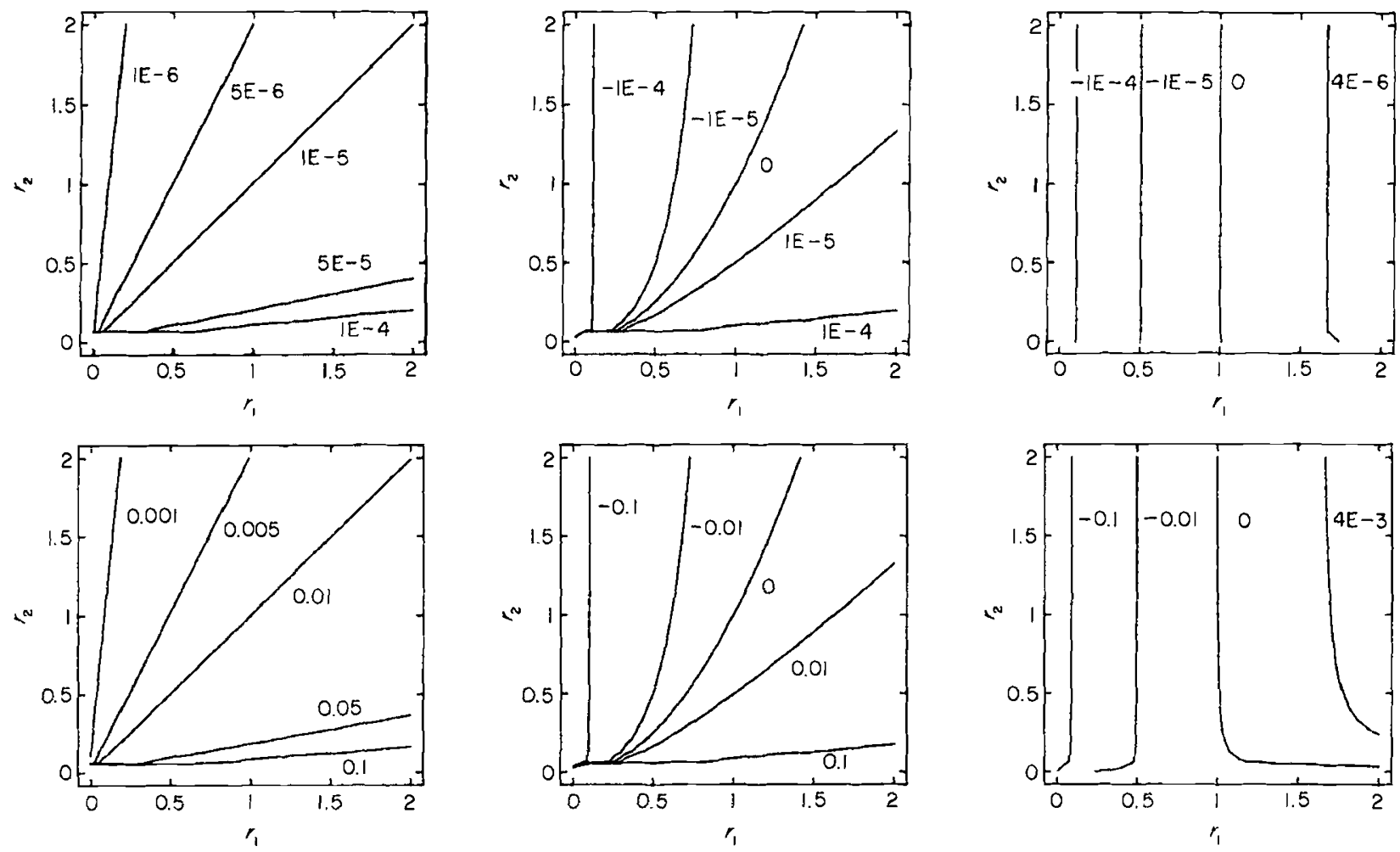

Fig. 1 Accuracy of the approximations given by equations (4) and (6) as a function of $r_{1}$ and $r_{2}$, given $p_{0}=10^{-5}$ (top row) and $p_{0}=10^{-2}$ (bottom row). In each row, the left contour plot shows the allele frequency $p_{1}$ after one generation of selection. The middle and right contour plots respectively show how well $u_{1}$ and $l_{1}$ approximate $p_{1}$ as measured by $\left(u_{1}-p_{1}\right) / p_{1}$ and $\left(l_{1}-p_{1}\right) / p_{1}$. 
value of $p_{1}$ as a function of $r_{1}$ and $r_{2}$, when $p_{0}=10^{-5}$. This is the surface to be approximated. To the right are contour plots of the accuracy with which $u_{1}$ and $l_{1}$ respectively approximate $p_{1}$. Here accuracy is measured by $\left(u_{1}-p_{1}\right) / p_{1}$ or $\left(l_{1}-p_{1}\right) / p_{1}$, so that a contour line labelled $-10^{-5}$ would indicate values of $r_{1}$ and $r_{2}$ for which the approximation underestimates $p_{1}$ by a factor of $10^{-5}$, or 0.001 per cent. The lower set of plots provide similar information for the case in which $p_{0}=0.01$.

Note that for all $p_{0}$, the first approximation $u_{1}$ equals $p_{1}$ along the line $r_{1}^{2}=r_{2}$ and underestimates or overestimates the actual allele frequency above or below that line, thereby always providing a strict bound. The accuracy is greater near this line, and varies systematically with $p_{0}$. In particular, when $p_{0}=10^{-5}, u_{1}$ is itself within a fraction $10^{-5}(0.001$ per cent $)$ of $p_{1}$ inside a large and broadening band about the line, and within a fraction $10^{-4}$ for essentially all values of the selection coefficients that were considered. However, when $p_{0}$ is increased 1000 -fold, so is the error. Therefore, for any $p_{0}, u_{1}$ is within a fraction $p_{0}$ of $p_{1}$ for moderate values of $r_{1}$ and $r_{2}$, and within a larger fraction $10 p_{0}$ for essentially all other $r_{1}$ and $r_{2}$.

The second approximation (right-hand plots) has similar properties. The error is 0 along a line (here a hyperbola) in the $\left(r_{1}, r_{2}\right)$ plane, and is smallest for values of $r_{1}$ and $r_{2}$ near this line. One important difference, however, is that because of the orientation of the hyperbola, unless $r_{2}$ is small the accuracy essentially depends only on $r_{1}$ and $p_{0}$, increasing as $r_{1} \rightarrow 1$ and as $p_{0} \rightarrow 0$. A comparison of the plots with $p_{0}=10^{-5}$ and $p_{0}=0.01$ shows that $l_{1}$ is within a fraction $p_{0}$ of the true value provided $r_{1}>0.5$, and within $10 p_{0}$ for essentially all $r_{1}>0$. This second approximation is therefore comparable to the first in accuracy, though for a larger region in the $\left(r_{1}, r_{2}\right)$ plane. It is also comparable in behaviour, as for any given fitness values the accuracy increases roughly 10 -fold for each 10 -fold reduction in $p_{0}$. One consequence is that when $p$ is increasing from small values, neither approximation will suffer from the compounding of large errors incurred when $p$ was small.

Figure 2 illustrates these points in the special case of complete dominance. Here, $p_{1}$ is always strictly bounded between $u_{1}$ and $l_{1}$, with the order depending on whether $r_{2}>1$ or $<1$. Both approximations are most accurate when $r_{2} \rightarrow 1$ and $p_{0} \rightarrow 0$. There is an important difference between them, however; when $r_{2}>0.5$, the first approximation $u_{1}$ is within a fraction $p_{0}$ of the true value of $p_{1}$ while the second, $l_{1}$, is within the smaller fraction $p_{0}^{2}$. Therefore, near $r_{1}=1$, the second approximation increases 100-fold in accuracy with each 10 -fold reduction in $p_{0}$, increasing from four-decimal place accuracy $(0.01$ per cent) when $p_{0}=10^{-2}$ to ten-decimal place accuracy when $p_{0}=10^{-5}$. Clearly, the second approximation is superior when $r_{1}$ is near 1 and $r_{1}^{2}$ is not near $r_{2}$ (near-full dominance), while the first approximation is particularly accurate when $r_{1}^{2}$ is near $r_{2}$ (approximately multiplicative effects).

Figure 3 illustrates the accuracy of both estimates after 10 generations of selection for all $r_{1}, r_{2} \leq 2$, starting with $p_{0}=10^{-5}$. Note that both $u_{10}$ and $l_{10}$ are still within $10^{-4}(0.01$ per cent $)$ of the actual value almost everywhere that $r_{2}>0.5$ and $r_{2}>r_{1}$, and $l_{10}$ is within 4 per cent provided only $r_{2}>0.5$. Both remain accurate, therefore, where there is weak or moderate selection for or against $A_{1}$, and strong selection against it. They are less accurate when there is strong selection favouring the allele $\left(r_{1}\right.$ and $r_{2}$ small, $p_{10}$ close to 1$)$, and when there is strong heterosis $\left(r_{1}\right.$ large, $r_{2}$ small, $p_{n}$ converging on an intermediate value).
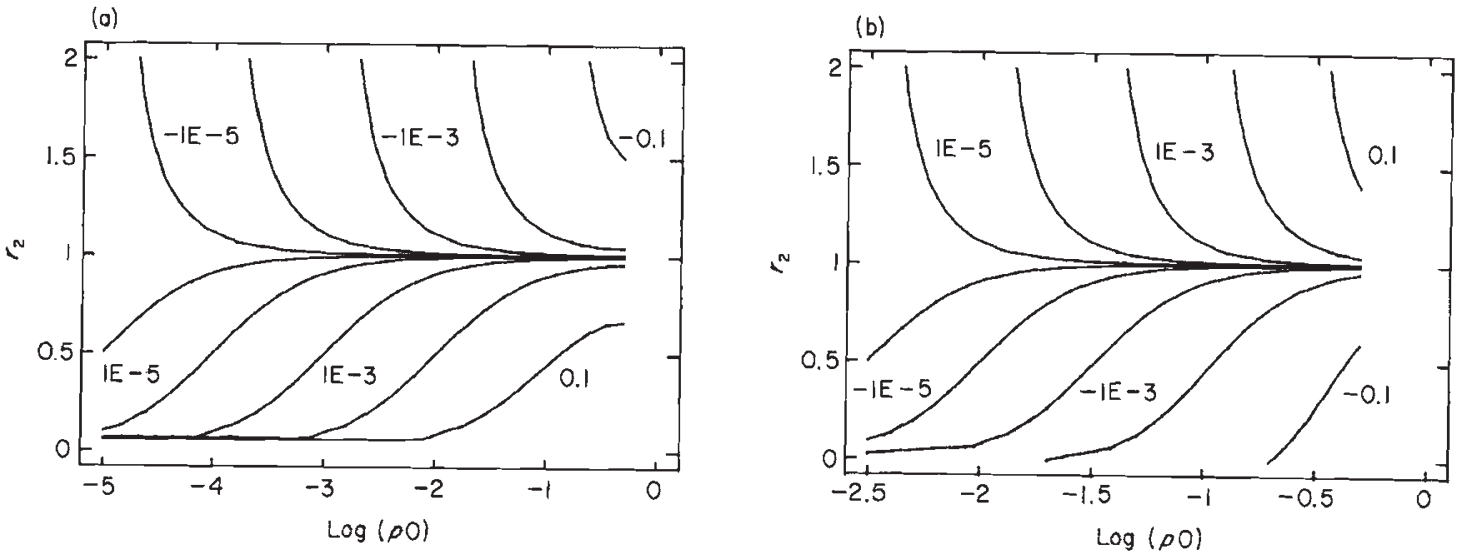

Fig. 2 Accuracy of the approximations given by equations (4) and (6) when there is complete dominance $\left(r_{1}=1\right)$, as a function of $p_{0}$ and $r_{2}$. As before, accuracy is measured by $\left(u_{1}-p_{1}\right) / p_{1}(\mathrm{a})$ and $\left(l_{1}-p_{1}\right) / p_{1}(\mathbf{b})$. 

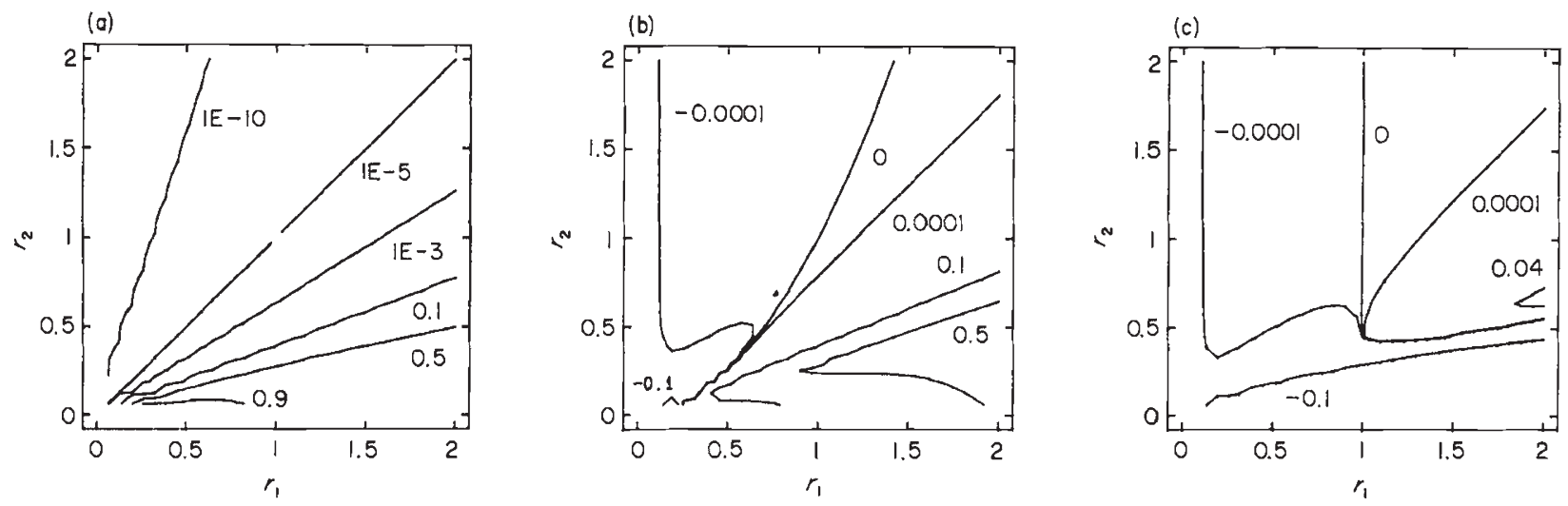

Fig. 3 Accuracy of the approximations given by equations (5) and (7) after 10 generations of selection, starting at $p_{0}=10^{-5}$, as a function of $r_{1}$ and $r_{2}$. (a) Shows the allele frequency $p_{10}$, while (b) and (c) respectively show how well $u_{10}$ and $l_{10}$ approximate $p_{10}$, as measured by $\left(u_{10}-p_{10}\right) / p_{10}$ and $\left(l_{10}-p_{10}\right) / p_{10}$.

\section{Approximations to $n$}

The approximations (5) and (7) above can be manipulated to yield estimates of the number of generations $n$ required for $\mathrm{A}_{1}$ to change in frequency from $p_{0}$ to some specified value $p_{n}$, given $r_{1}$ and $r_{2}$.

$$
\begin{aligned}
& n_{u}=\frac{1}{\ln (R)} \ln \left[\frac{p_{0}\left(1-p_{n}\right)}{\left.p_{n} 1-p_{0}\right)}\right] \\
& n_{l}=\frac{1}{\ln (R)} \ln \left[\frac{p_{0}\left(1-2 p_{n}\right)}{p_{n}\left(1-2 p_{0}\right)}\right]
\end{aligned}
$$

As before, the second approximation is restricted to the case in which $p_{0}$ and $p_{n}<0.5$.

Note that whenever $u_{n}$ or $l_{n}$ provides a bound on the amount of allele frequency change in $n$ generations, then $n_{u}$ or $n_{l}$ will place the opposite bound on the number of generations required to achieve that change. Under certain conditions the two approximations can be combined to bracket the exact solution. In particular, if $1 \geq r_{1}^{2}>r_{2}$, then since $u_{n}$ and $l_{n}$ respectively overestimate and underestimate the rate of increase in $p$, the actual number of generations required for any specified change in $p$ will be bracketed by $n_{u}<n<n_{l}$. If the direction of selection is reversed and the fitness values obey $1 \leq r_{1}^{2}<r_{2}$, then $u_{n}$ overestimates the rate of decline in $p$, and $l_{n}$ underestimates it, and again the actual $n$ for any specified change is bounded by $n_{u}<n<n_{l}$.

The accuracy of these two approximations is illustrated in Table 1, which presents the exact number of generations required for specific changes in $p$ as calculated by iteration, and the estimates obtained with equations (9) and (10). In all but one case, the selection regimes considered satisfy the requirements that $n$ is bounded on either side by $n_{u}<n<n_{l}$, although often the error is so small as to vanish on rounding off to the nearest generation. Both approximations are essentially exactly correct whenever the beginning and final allele frequencies do not exceed 0.1 , regardless of whether the $A_{1}$ allele is fully or incompletely dominant, favoured or opposed in selection. In some cases, the accuracy remains high even at higher frequencies, with the best predictor depending on the selection coefficients. Thus when $r_{1}=1$ and $r_{2}=0.99$, there is weak selection favouring the dominant allele, and the second approximation is expected to be more accurate. In fact, it predicts that 1036 generations are required for $p$ to increase from $10^{-5}$ to 0.2 , and is high by only three generations, whereas the first approximation is low by 24. Similarly, in the two cases in which $r_{1}^{2}$ is near $r_{2}$, the second approximation is close but the first gives the exact solution over the entire range of allele frequencies considered: 205 generations to increase from $10^{-5}$ to 0.4 when $r_{1}=0.95$ and $r_{2}=0.90$, and 239 generations to decrease from 0.4 to $10^{-5}$ when $r_{1}=1.05$ and $r_{2}=1.10$.

\section{Alternative approximations}

The approximations first offered here are intermediate in complexity and accuracy to others already in the literature. At one extreme is the estimate $p_{n} \approx p_{0} / R^{n}$, obtained by setting all terms in both $p_{0}$ and $p_{0}^{2}$ equal to 0 in the formula for $p_{1} / p_{0}$ (e.g. Jacquard, 1974). Although crude, this returns accurate solutions when $p_{0}$ and $p_{n}$ are both small. The corresponding estimate for the number of generations required to pass from $p_{0}$ to $p_{n}$ is $n \approx \ln \left(p_{0} / p_{n}\right) / \ln (R)$.

At the other extreme are quite complicated formulas obtained from differential equation approximations to the difference equation (1). Haldane (1926) obtained an approximate solution to (1) which he had written 
Table 1 Number of generations required for the $\mathrm{A}_{1}$ allele to change in frequency from $p_{0}$ to $p_{n}$ given $r_{1}$ and $r_{2}$ as specified below, as calculated exactly be iteration of equation (1), and as estimated by equation (9) $n_{u}$ and (10) $n_{l}$. Note that in all but the last case, the fitness values satisfy the conditions that ensure $n_{u} \leq n_{\text {exact }} \leq n_{l}$

\begin{tabular}{|c|c|c|c|c|c|c|c|c|c|}
\hline$p_{0} \rightarrow p_{n}$ & $10^{-5} \rightarrow 10^{-2}$ & $10^{-2} \rightarrow 0.1$ & $0.1 \rightarrow 0.2$ & $0.2 \rightarrow 0.4$ & $p_{0} \rightarrow p_{n}$ & $0.4 \rightarrow 0.2$ & $0.2 \rightarrow 0.1$ & $0.1 \rightarrow 10^{-2}$ & $10^{-2} \rightarrow 10^{-5}$ \\
\hline \multicolumn{5}{|c|}{$r_{1}=1, r_{2}=0.99$} & \multicolumn{5}{|c|}{$r_{1}=1, r_{2}=1.01$} \\
\hline$n_{u}$ & 688 & 239 & 81 & 98 & $n_{u}$ & 99 & 81 & 241 & 695 \\
\hline$n_{\text {exact }}$ & 689 & 249 & 95 & 139 & $n_{\text {exact }}$ & 140 & 95 & 251 & 696 \\
\hline$n_{l}$ & 689 & 249 & 98 & 178 & $n_{l}$ & 180 & 99 & 252 & 696 \\
\hline \multicolumn{5}{|c|}{$r_{1}=1, r_{2}=0.90$} & \multicolumn{5}{|c|}{$r_{1}=1, r_{2}=1.10$} \\
\hline$n_{u}$ & 66 & 23 & 8 & 9 & $n_{u}$ & 10 & 9 & 25 & 73 \\
\hline$n_{\text {exact }}$ & 66 & 24 & 9 & 13 & $n_{\text {exact }}$ & 15 & 10 & 26 & 73 \\
\hline$n_{l}$ & 66 & 24 & 9 & 17 & $n_{l}$ & 19 & 10 & 26 & 73 \\
\hline \multicolumn{5}{|c|}{$r_{1}=0.95, r_{2}=0.90$} & \multicolumn{5}{|c|}{$r_{1}=1.05, r_{2}=1.10$} \\
\hline$n_{u}$ & 128 & 44 & 15 & 18 & $n_{u}$ & 21 & 17 & 52 & 149 \\
\hline$n_{\text {exact }}$ & 128 & 44 & 15 & 18 & $n_{\text {exact }}$ & 21 & 17 & 52 & 149 \\
\hline$n_{l}$ & 128 & 46 & 18 & 33 & $n_{l}$ & 39 & 21 & 54 & 149 \\
\hline
\end{tabular}

$\left.U_{1}-U_{0}=U_{0}\left[K U_{0}-K+k\right] /\left[(1-K) U_{0}+1-k\right)\right]$,

where $U=p /(1-p), \quad K=1-r_{1} \quad$ and $\quad k=1-r_{2}$. Assuming selection to be weak, he approximated the denominator by $U_{0}+1$ and the difference $U_{1}-U_{0}$ by $\mathrm{d} U / \mathrm{d} n$ to yield

$$
n=\frac{1}{r_{1}-r_{2}} \ln \left[\left(\frac{p_{n}}{p_{0}}\right)\left(\frac{1-p_{0}}{1-p_{n}}\right)^{1 /\left(R_{1}+1\right)}\left(\frac{R_{1} p_{0}+1}{R_{1} p_{n}+1}\right)^{R_{1} /\left(R_{1}+1\right)}\right],
$$

where $R_{1}=\left(1-2 r_{1}+r_{2}\right) /\left(r_{1}-r_{2}\right)$. Wright (1931) obtained this answer multiplied by $r_{2}$ from a differential equation approximation in $p$,

$$
\mathrm{d} p / \mathrm{d} n \approx p_{1}-p_{0} \approx\left[1 / r_{2}\right] p(1-p)\left[\left(1-2 r_{1}+r_{2}\right) p+r_{1}-r_{2}\right] \text {, }
$$

where the denominator had been set equal to 1 after assuming weak selection. In the special case of complete dominance, these formulas simplify somewhat (e.g. Haldane, 1924), and Haldane (1932) even obtained an exact solution, although in the form of an infinite series which itself must be approximated. All these estimates are extremely accurate over a wide range of parameter values and initial conditions. However, they are unwieldy and therefore difficult to calculate, interpret, and incorporate into larger models. Moreover, unlike the approximations introduced here, they cannot by manipulated to yield estimates of $p_{n}$ that are explicit functions of $p_{0}, r_{1}, r_{2}$ and $n$.

\section{Discussion}

To the extent that equations (5) and (7) provide accurate descriptions of the effect of selection on allele frequencies, several additional quantitative relations can be noted. First, it is clear from either equation that $n$ generations of selection with fitness $1, r_{1}$, and $r_{2}$ is approximately equal to one generation of selection with fitnesses $1, r_{1}^{n}, r_{2}^{n}$. (It is slightly more or less, according to whether $r_{1}^{2}<r_{2}$.) This generalizes a point by $\mathrm{Li}(1959)$, who noted the exact equivalence when $r_{1}^{2}=r_{2}$. Alternatively, one generation of selection with fitnesses $1, r_{1}$, and $r_{2}$ is equivalent to $x$ generations at 1 , $r_{1}^{*}$, and $r_{2}^{*}$, where $x$ is defined by $\left(r_{2} / r_{1}\right)=\left(r_{2}^{*} / r_{1}^{*}\right)^{x}$. Thus for example the degree to which being recessive protects deleterious rare alleles can be quantified. If the fitnesses are $1, r_{1}=1.98$ and $r_{2}=2$, where the deleterious allele is effectively protected in heterozygote form, then 29 generations of selection are required to effect the same decrease in $p$ as 1 generation of selection without dominance, $r_{1}=1.5$.

Finally, while equations (4) and (6) are rational approximations to (1), in that they are ratios of polynomials, they were not obtained through the normal machinery of rational approximation theory (e.g. Newman, 1979). The usual approach to approximate a function such as (1) over an interval is to select a class of approximating functions, for example, polynomials (e.g. Taylor's series) or trigonometric functions (Fourier expansions), and a measure of distance between the function and its approximation (absolute value, squared deviation, etc.) to be minimized. Once the problem is defined, one considers the existence, uniqueness, characteristics, and construction of its solution, which consists of the coefficients that achieve a good or best fitting function of the class, as measured by the metric (e.g. Rice, 1964; Lorentz, 1966).

The approximations proposed here were selected instead on the basis of three considerations. First it was necessary to approximate equation (1) for all $r_{1}$ and $r_{2}$, and not merely a specific case such as when $r_{1}=0.95$ 
and $r_{2}=0.9$. This could be viewed as a multivariate approximation problem in $r_{1}, r_{2}$, and $p_{0}$. Second, the result had to be a difference equation in $p$ that could be solved in closed form, as the goal is to find $p_{n}$, not merely $p_{1}$. Other approximations that were considered either could not be solved or were less accurate than the two selected. Third, the accuracy had to be greater at lower values of $p_{0}$ : an error of $10^{-2}$ or even $10^{-4}$ when $p_{0}=10^{-5}$ is unacceptable and, when iterated, would grow even larger. The identification of the 'best' rational approximation to equation (1) under these constraints may be a non-trivial problem, and might not provide any further advantage over the 'good' approximations given by equations (4) and (6).

The approximations introduced here satisfy all three criteria above, and offer several additional advantages as well. In particular, they provide simple, explicit, and transparent estimates for either $n$ or $p_{n}$, unlike the more complex approximations available which can only estimate $n$. In addition, these estimates are especially accurate (certainly within measurement error) for fitness values near 1 that are most often encountered. Finally, the first approximation always places a strict bound on $p_{n}$ or $n$, and the second approximation often provides the other bound. Therefore, one can not only estimate $p_{n}$ or $n$, but also know the sign of the error and, in some cases, its maximum value. The more-complex estimates by Haldane and Wright are available when greater accuracy is required, especially at higher allele frequencies; however, these equations may be unwieldy for some applications, for example, as components in models with population dynamics, and they cannot by manipulated to yield $p_{n}$ as an explicit function of $p_{0}$.

\section{References}

FELdMAN, M. W. (ed.) 1989. Mathematical Evolutionary Theory. Princeton University Press, Princeton.

HALDANE, J. B. S. 1924. A mathematical theory of natural and artificial selection. Part I. Trans. Camb. Phil. Soc., 23, 19-41.

HALDANE, J. B. S. 1926. A mathematical theory of natural and artificial selection. Part III. Proc. Camb. Phil. Soc., 23, 363-372.

HALDANE, J. B. S. 1932a. The Causes of Evolution. Longman, London.

HALDANE, J. B. S. 1932 b. A mathematical theory of natural and artificial selection. Part IX. Rapid selection. Proc. Camb. Phil. Soc., 28, 244-248.

JACQUARD, A. 1974. The Genetic Structure of Populations. Springer-Verlag, New York.

L1, C. C. 1959 . Notes on relative fitness of genotypes that forms a geometric progression. Evolution, 13, 564-567.

L1, C. C. 1976. First Course in Population Genetics. Boxwood Press, Pacific Grove, CA.

LORENTZ, G. G. 1966. Approximation of Functions. Holt, Rinehart and Winston, New York.

NEWMAN, D. J. 1979. Approximation with Rational Functions. American Mathematical Society, Providence, Rhode Island.

RICE, J. R. 1964. The Approximation of Functions. Addison Wesley, Reading, MA.

WRIGHT, S. 1931. Evolution in Mendelian populations. Genetics, 16, 97-159. 